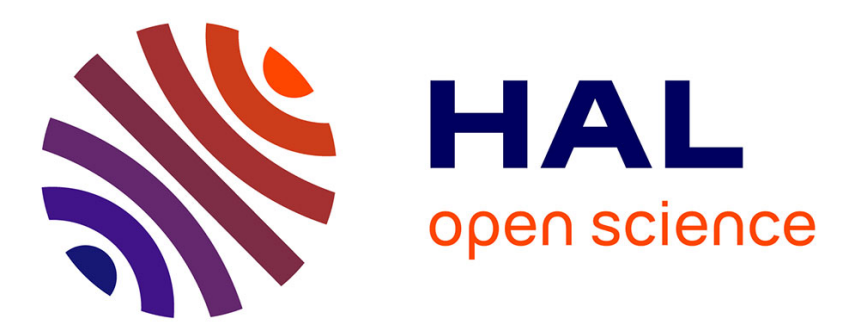

\title{
Infinite disorder and correlation fixed point in the Ising model with correlated disorder
}

Christophe Chatelain

\section{To cite this version:}

Christophe Chatelain. Infinite disorder and correlation fixed point in the Ising model with correlated disorder. The European Physical Journal. Special Topics, 2017, 226, pp.805. 10.1140/epjst/e201660332-9 . hal-01383193

\section{HAL Id: hal-01383193 \\ https://hal.science/hal-01383193}

Submitted on 18 Oct 2016

HAL is a multi-disciplinary open access archive for the deposit and dissemination of scientific research documents, whether they are published or not. The documents may come from teaching and research institutions in France or abroad, or from public or private research centers.
L'archive ouverte pluridisciplinaire HAL, est destinée au dépôt et à la diffusion de documents scientifiques de niveau recherche, publiés ou non, émanant des établissements d'enseignement et de recherche français ou étrangers, des laboratoires publics ou privés. 


\title{
Infinite disorder and correlation fixed point in the Potts model with correlated disorder
}

\author{
C. Chatelain ${ }^{1}$
}

Groupe de Physique Statistique, Département P2M, Institut Jean Lamour (CNRS UMR 7198), Université de Lorraine, France, e-mail: christophe.chatelain@univ-lorraine.fr

\begin{abstract}
Recent Monte Carlo simulations of the $q$-state Potts model with a disorder displaying slowly-decaying correlations reported a violation of hyperscaling relation caused by large disorder fluctuations and the existence of a Griffiths phase, as in random systems governed by an infinite-disorder fixed point. New simulations, directly made in the limit of an infinite disorder strength, are presented. The magnetic scaling dimension is shown to correspond to the correlated percolation fixed point. The latter is shown to be unstable at finite disorder strength but with a large cross-over length which is not accessible to Monte Carlo simulations.
\end{abstract}

\section{Introduction}

Disorder is present in all experimental systems but it does not lead to the same consequences. The study of its influence on phase transitions is of particular interest because even a weak disorder can induce drastic changes. First-order phase transitions are softened and may even become continuous if the disorder is sufficiently strong or the space dimension equal to two $[1,2]$. When the transition of the pure system is already continuous, its critical behavior is changed by the introduction of disorder when the random fluctuations grow faster with the system size than energy fluctuations [3]. The critical behavior of the random system is then governed by a new Renormalization Group ( $\mathrm{RG}$ ) fixed point. The fixed point of the pure model, still present, is unstable but may cause a cross-over at weak disorder. A clear example of a change of universality class upon the introduction of disorder is provided by the two-dimensional 3 or 4 state Potts model, a generalization of the celebrated Ising model. When exchange couplings are made random, new critical exponents were measured numerically [4-6] and shown to be in agreement with RG calculations [7-10]. Experimentally, the phase transition of the 4-state Potts model can be realized by the order-disorder transition of atoms binded on a surface offering four inequivalent adsorption sites in presence of oxygen impurities [11].

In the above-mentioned Potts models, the disorder is assumed to be quenched and to consist in uncorrelated random couplings. However, at some point of the evolution of the system, in particular during its preparation, or at a much larger time scale, impurities may diffuse in the sample and thermalize. If there exists an interaction between them, their equilibrium configurations may display long-range correlations. Renormalization Group studies of the $\phi^{4}$ model showed that disorder correlations 
with an exponential or a fast algebraic decay do not affect the critical behavior. In contrast, slowly decaying disorder correlations, slower than $r^{-2 / \nu}$ where $\nu$ is the correlation length exponent of the pure model, bring the system towards a new RG fixed point, distinct from the pure one and from the one associated to uncorrelated or shortrange disorder [12]. These predictions were confirmed by Monte Carlo simulations of the Ising model $[13,14]$ and by RG calculations directly in dimension $d=2[15]$.

In the recent years, Monte Carlo simulations of the Potts model with very slowly decaying correlations between the random couplings led to results that do not fit in this well-accepted picture $[16,17]$. Several intriguing features, already known in other models but not in the short-range random Potts model, were observed. First, the magnetic susceptibility displays a power-law divergence with the lattice size, not only at the critical point, but in a finite range of temperatures around the self-dual critical point. Such a region of the phase diagram, known as a Griffiths phase [18], was first observed in the McCoy-Wu model $[20,21]$ or, equivalently in the extreme anisotropic limit, in the random quantum Ising chain in a transverse field [22]. It is explained as the consequence of the existence, though with an exponentially small probability, of macroscopically large clusters with a high concentration of strong (resp. weak) couplings. These clusters can order earlier (later) than the rest of the system, i.e. already in the paramagnetic (ferromagnetic) phase [19]. Second, a violation of the hyperscaling relation $(\gamma+2 \beta) / \nu=d$ was reported. Such a violation exists in pure models above their upper critical dimension, and, for different reasons, in the classical 3D Ising model in a random field [23]. In the latter, the origin of the hyperscaling violation is found in the different algebraic decay of typical and average spin-spin correlations. The same mechanism was proposed in the long-range random Potts model. Third, the new universality class does not seem to depend on the number of states $q$ of the Potts model while in the case of uncorrelated disorder a dependence of the magnetic scaling dimension $x_{\sigma}=\beta / \nu$ on $q$ was unambiguously observed numerically. The independence on the number of states $q$ is also found in the 1D random quantum Potts model [24]. Moreover, the numerical estimates of the critical exponents are remarkably stable with the disorder strength and no sign of cross-over could be distinguished.

In this paper, the 2D classical Potts model with slowly-decaying disorder correlations is considered. In contrast to previous studies, the numerical calculations are performed in the limit of an infinite disorder. After a short presentation of the model, the magnetic scaling dimension is estimated numerically for different disorder correlations in section 3 . In section 4 , the relevance of a large but finite disorder is investigated. A conclusion follows.

\section{Definition of the model}

The $2 \mathrm{D}$ classical $q$-state Potts model is considered on the square lattice. The Hamiltonian is $[25]$

$$
-\beta \mathcal{H}=\sum_{(i, j) \in E} J_{i j} \delta_{s_{i}, s_{j}}, \quad s_{i} \in\{0, \ldots, q-1\}
$$

where the sum extends over the set $E$ of pairs of neighboring sites of the lattice. The Ising model is recovered when $q=2$. We are interested in the case where the exchange couplings are random and display algebraic correlations with the distance:

$$
\overline{J_{i j} J_{k l}}-\overline{J_{i j}} \overline{J_{k l}} \sim\left|\mathbf{r}_{i j}-\mathbf{r}_{k l}\right|^{-a} \text {. }
$$


In previous studies, it was found convenient to generate these coupling configurations by performing a Monte Carlo simulation of another lattice spin model, the isotropic Ashkin-Teller model. Its Hamiltonian [26]

$$
-\beta \mathcal{H}_{\mathrm{AT}}=\sum_{(i, j) \in E}\left[J_{\mathrm{AT}}\left(\sigma_{i} \sigma_{j}+\tau_{i} \tau_{j}\right)+K_{\mathrm{AT}} \sigma_{i} \sigma_{j} \tau_{i} \tau_{j}\right], \quad \sigma_{i}= \pm 1, \tau_{i}= \pm 1 .
$$

is invariant under the two global $\mathbb{Z}_{2}$ transformations:

$$
\sigma_{i} \longrightarrow-\sigma_{i}, \quad\left(\sigma_{i}, \tau_{i}\right) \longrightarrow\left(-\sigma_{i},-\tau_{i}\right)
$$

As a consequence, the phase diagram displays three phases: a mixed, or Baxter, phase where both symmetries are spontaneously broken, a ferromagnetic phase where the two Ising copies are ordered but not correlated between them and a paramagnetic phase. Magnetization and polarization

$$
M=\sum_{i \in V} \sigma_{i}, \quad P=\sum_{i \in V} \sigma_{i} \tau_{i}
$$

are order parameters for the phase transitions between these phases. $V$ is the set of lattice sites. Interestingly for our purpose, the two symmetries are simultaneously broken along a critical line which is known exactly by self-duality arguments [27]:

$$
\sinh 2 J_{\mathrm{AT}}=e^{-2 K_{\mathrm{AT}}}
$$

While the magnetic scaling dimension $x_{\sigma}=1 / 8$ is constant along the line, the polarization scaling dimension is given by

$$
x_{\sigma \tau}=\frac{1}{8-4 y}
$$

where the parametrization

$$
\cos \frac{\pi y}{2}=\frac{1}{2}\left[e^{4 K_{\mathrm{AT}}}-1\right]
$$

was introduced. Along the self-dual critical line, polarization-polarization correlations decay algebraically as

$$
\overline{\sigma_{i} \tau_{i} \sigma_{j} \tau_{j}} \sim\left|\mathbf{r}_{i}-\mathbf{r}_{j}\right|^{-2 x_{\sigma \tau}}
$$

while the average polarization density $\overline{\sigma_{i} \tau_{i}}$ vanishes.

The procedure to generate correlated random couplings for the Potts model is the following: Monte Carlo simulations of an auxiliary Ashkin-Teller model are performed at various points $y$ of its self-dual critical line. Statistically uncorrelated spin configurations are sampled by throwing away a number of Monte Carlo steps several times larger than the autocorrelation time. These spin configurations are transformed into coupling configurations:

$$
J_{i j}=\frac{J_{1}+J_{2}}{2}+\sigma_{i} \tau_{i} \frac{J_{1}-J_{2}}{2}, \quad \forall(i, j) \in E .
$$

On each site, one horizontal and one vertical coupling take the same value. This should not have any relevant effect on the physics at large distance. For each coupling configurations, a Monte Carlo simulation of the Potts model is performed. The construction of the random couplings (Eq. 10) ensures that the disorder correlations 

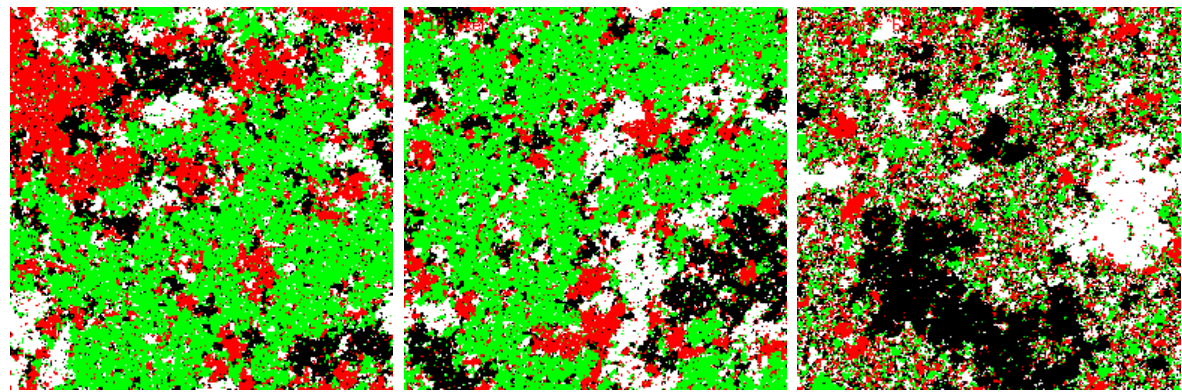

Fig. 1. Typical spin configurations of the critical 4-state Potts model with uniform coupling (left), uncorrelated random couplings (center) and correlated couplings (right). In the last two cases, the disorder strength is $J_{1} / J_{2}=4$.

will decay algebraically as the polarization-polarization correlations of the auxiliary Ashkin-Teller model. The exponent $a$ of disorder correlation is given by $a=2 x_{\sigma \tau}$. For the random Potts model, averages are computed over both thermal and disorder fluctuations:

$$
\overline{\langle X\rangle}=\frac{1}{\mathcal{Z}_{\mathrm{AT}}} \sum_{\{\sigma, \tau\}}\left[\frac{1}{\mathcal{Z}} \sum_{\{s\}} X[s] e^{\sum_{(i, j)} J_{i j}[\sigma, \tau] \delta_{s_{i}, s_{j}}}\right] e^{-\beta H_{\mathrm{AT}}[\sigma, \tau]}
$$

where the brackets stands for the average over thermal fluctuations for a given disorder configuration and the overline for the average over disorder.

Typical spin configurations of the Potts model at its self dual critical point are presented on figure 1. In contrast to the case of uncorrelated disorder, the typical spin configurations display coexisting paramagnetic and ferromagnetic clusters when random couplings are correlated. Paramagnetic (resp. ferromagnetic) clusters are favored by a high density of weak (resp. strong) couplings. Thermal fluctuations are essentially limited to the interior of each cluster and do not induce any significant fluctuation of their boundaries. Even though the disorder strength is relatively small ( $r=J_{1} / J_{2}=4$ in the configurations presented on the figure), the Potts model behaves as in the limit of infinite disorder. The spin configurations of the Potts model are highly correlated with the disorder realization and, subsequently with the polarization configuration of the auxiliary Ashkin-Teller model. The coexistence of paramagnetic and ferromagnetic clusters does not imply that the random Potts model undergoes a first-order phase transition. The Ashkin-Teller model being critical and symmetric under polarization reversal, clusters of all sizes, both with strong and weak couplings, are present in each disorder configuration. Each cluster will undergo a sharp ferromagnetic-to-paramagnetic transition at a different temperature so the average magnetization curve will be much smoother than the curve of a single cluster. The argument is similar to the Imry-Wortis criterion [1]. More important, the criticality of the Ashkin-Teller model ensures that, in the thermodynamic limit, a percolating cluster is present in any polarization configuration. As a consequence, a spanning cluster of either strong or weak couplings is present in each disorder configuration. In the former case, long-range ferromagnetic order will be induced in the Potts model.

\section{Magnetic scaling dimension}

In this section, the magnetic scaling dimension of the long-range random Potts model is estimated in the infinite-disorder limit. Magnetization is more easily computed in 
the case of the Ising model, i.e. $q=2$. The Hamiltonian becomes, up to a constant term,

$$
-\beta H=\sum_{(i, j)} K_{i j} s_{i} s_{j}, \quad s_{i} \in\{+1,-1\}
$$

where $K_{i j}=J_{i j} / 2$ is half of the coupling of the original Potts model. For a given disorder realization, the partition function reads

$$
\mathcal{Z}[K]=\sum_{\{s\}} e^{\sum_{(i, j)} K_{i j} s_{i} s_{j}}
$$

and the average square magnetization is computed as

$$
\left\langle M^{2}\right\rangle=\frac{1}{\mathcal{Z}[K]} \sum_{\{s\}}\left(\sum_{i} s_{i}\right)^{2} e^{\sum_{(i, j)} K_{i j} s_{i} s_{j}}
$$

The average over disorder leads to

$$
\overline{\left\langle M^{2}\right\rangle}=\int_{\mathbb{R}|E|} \frac{1}{\mathcal{Z}[K]} \sum_{\{s\}}\left(\sum_{i} s_{i}\right)^{2} e^{\sum_{(i, j)} K_{i j} s_{i} s_{j}} \wp[K] \prod_{(i, j)} d K_{i j}
$$

As discussed in the previous section, the disorder configurations $\left\{K_{i j}\right\}$ are either random or determined from the spin configurations $\{\sigma, \tau\}$ of an auxiliary spin model, the Ashkin-Teller model. In both cases, the random couplings can take only two values, $K_{1}$ or $K_{2}$.

In the limit of an infinite disorder, $K_{1} \rightarrow+\infty$ and $K_{2} \rightarrow 0$, neighboring spins connected by a coupling $K_{1}$ are frozen in the same state. The partition function is reduced to the number of ways that the connected clusters of the graph formed by the strong bonds can be decorated with Ising spins. Given a disorder configuration $\left\{K_{i j}\right\}$, the partition function tends towards

$$
\mathcal{Z}\left[K_{i j}\right]=\sum_{\{s\}} e^{\sum_{(i, j)} K_{i j} s_{i} s_{j}} \sim e^{\left|E_{1}\right| K_{1}} \sum_{\{s\}} \prod_{(i, j) \in E_{1}} \delta_{s_{i}, s_{j}}=e^{\left|E_{1}\right| K_{1}} \times 2^{C\left[E_{1}\right]}
$$

where $E_{1}=\left\{(i, j) / K_{i j}=K_{1}\right\} \subset E$ is the set of strong bonds, $\left|E_{1}\right|=\sum_{(i, j)} \delta\left(K_{i j}-\right.$ $K_{1}$ ) the number of strong bonds and $C\left[E_{1}\right]$ the number of connected clusters in the graph $E_{1}$ formed by the strong bonds. The free energy is

$$
f\left[K_{i j}\right]=-\ln \mathcal{Z}\left[K_{i j}\right]=-\left|E_{1}\right| K_{1}-C\left[E_{1}\right] \ln 2
$$

where $\left|E_{1}\right| K_{1}$ is an energy term while $C\left[E_{1}\right] \ln 2$ is the entropy associated to the number of spin decorations of the connected clusters of the graph $E_{1}$. Note also when $K_{i j}$ is determined by $\sigma_{i} \tau_{i},\left|E_{1}\right|$ and $C\left[E_{1}\right]$ are functions of the Ashkin-Teller spin configurations $\{\sigma, \tau\} .\left|E_{1}\right|$ is related to the total polarization $P=\sum_{i \in V} \sigma_{i} \tau_{i}$ by $\left|E_{1}\right|=|V|+P$ where $|V|$ is the number of lattice sites.

The second moment of magnetization is, for a given disorder configuration,

$$
\begin{aligned}
\left\langle M^{2}\right\rangle_{E_{1}} & =\frac{1}{\mathcal{Z}\left[K_{i j}\right]} e^{\left|E_{1}\right| K_{1}} \sum_{\{s\}}\left(\sum_{i} s_{i}\right)^{2} \prod_{(i, j) \in E_{1}} \delta_{s_{i}, s_{j}} \\
& \sim 2^{-C\left[E_{1}\right]} \sum_{\{s\}}\left(\sum_{i} s_{i}\right)^{2} \prod_{(i, j) \in E_{1}} \delta_{s_{i}, s_{j}}
\end{aligned}
$$




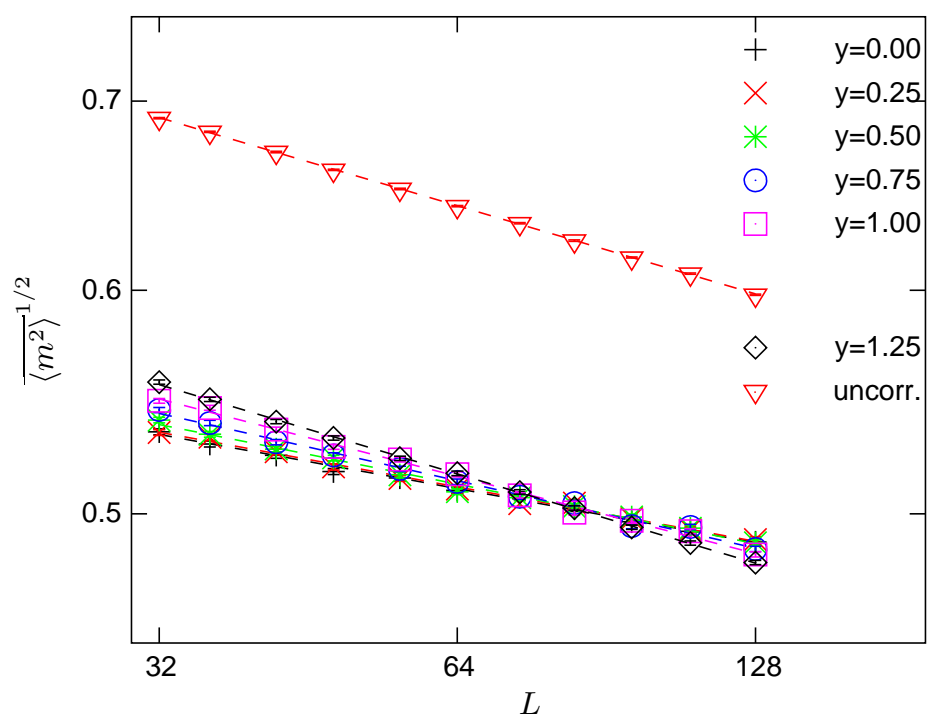

Fig. 2. Finite-size scaling of the second moment of the magnetization density ${\overline{\left\langle m^{2}\right\rangle}}^{1 / 2}$ of the $2 \mathrm{D}$ Ising model in the limit of infinite disorder. The different symbols correspond to the Monte Carlo data for different disorder correlations. The parameter $y$ is given in the legend. The case of uncorrelated disorder is also plotted and denoted 'uncorr.' in the legend. Errors bars are plotted. The dashed lines are the power-law fits.

Numerically, this quantity is evaluated in the following way: first, the graph of strong bonds is identified and the connected clusters are labeled. The number of sites of each clusters is determined. Since spins are frozen in the same state in a cluster, the total magnetization reads

$$
M\left[E_{1}\right]=\sum_{\alpha=1}^{C\left(E_{1}\right)} N_{\alpha} s_{\alpha}
$$

where $N_{\alpha}$ is the number of spins in the $\alpha$-th cluster and $s_{\alpha} \in\{+1,-1\}$ is the value of all spins in this cluster. When averaged over all possible spin configurations, the total magnetization $M$ vanishes. In contrast

$$
M^{2}\left[E_{1}\right]=\left(\sum_{\alpha=1}^{C\left(E_{1}\right)} N_{\alpha} s_{\alpha}\right)^{2}=\sum_{\alpha} N_{\alpha}^{2}+\sum_{\alpha, \beta \neq \alpha} N_{\alpha} N_{\beta} s_{\alpha} s_{\beta}
$$

and, since spins are uncorrelated, i.e. $\left\langle s_{\alpha} s_{\beta}\right\rangle=0$ when $\alpha \neq \beta$, the last term vanishes and the second moment reads

$$
\left\langle M^{2}\right\rangle_{E_{1}}=\sum_{\alpha} N_{\alpha}^{2}
$$

Note that the prefactor $2^{-C\left[E_{1}\right]}$ appearing in Eq. 18 is precisely the number of spin decorations of the clusters so that it cancels with the sum $\sum_{\{s\}}$. Finally, the disorder average $\overline{\left\langle M^{2}\right\rangle}$ is performed.

On figure 2, the Monte Carlo estimate of the second moment of the magnetization density ${\overline{\left\langle m^{2}\right.}}^{1 / 2}$ is plotted versus the lattice size $L$ for different values of $y$ and for uncorrelated disorder. As discussed above, each value of $y$ corresponds to a correlated disorder with a different algebraic decay of disorder correlations. The data have been 
Table 1. Numerical estimates of the magnetic scaling dimension $\beta / \nu$ for correlated disorder $(y \in[0 ; 1.25])$ and for uncorrelated disorder ('uncorr.') as discussed in section 3 . The exponent $a$ of the algebraic decay of disorder correlation is given in the second column. The last two columns correspond to the Monte Carlo estimates of the exponent $\omega$ of the algebraic decay of the first-order corrections $C_{1}$ and $C_{2}$ of the square magnetization (see section 4 for details).

\begin{tabular}{lllll}
\hline$y$ & $a$ & $\beta / \nu$ & Correction & \\
\hline 0.00 & 0.25 & $0.064(2)$ & $0.135(3)$ & $0.125(3)$ \\
0.25 & 0.286 & $0.064(2)$ & $0.144(3)$ & $0.134(3)$ \\
0.50 & 0.333 & $0.070(2)$ & $0.170(3)$ & $0.152(3)$ \\
0.75 & 0.4 & $0.079(2)$ & $0.190(3)$ & $0.167(3)$ \\
1.00 & 0.5 & $0.091(2)$ & $0.194(3)$ & $0.166(3)$ \\
1.25 & 0.667 & $0.1050(8)$ & $0.192(3)$ & $0.161(3)$ \\
uncorr. & 2 & $0.1037(4)$ & $0.786(2)$ & $0.788(3)$ \\
\hline
\end{tabular}

averaged over 100.000 disorder configurations. While random couplings belonging to the same disorder configuration are correlated, there is no correlation between different disorder configurations. Therefore, $\left\langle m^{2}\right\rangle$ are uncorrelated random variables and the error on $\overline{\left\langle m^{2}\right\rangle}$ can be estimated from the mean square deviation.

The data show a nice algebraic behavior over the full range of lattice sizes considered. The associated critical exponents $\beta / \nu$ are reported in table 1 . They are in good agreement with the values previously obtained with more standard Monte Carlo simulations at finite disorder strength $r=K_{1} / K_{2}$ [17]. This supports the assumption of a critical behavior governed by an infinite-randomness fixed point. In contrast, the estimate of $\beta / \nu$ for uncorrelated disorder $(0.1037(4))$ is incompatible with the expected exponent for the $2 \mathrm{D}$ random Ising model. Since randomness is marginally irrelevant in this case, the exponent $\beta / \nu=1 / 8$ of the pure fixed point was expected. However, we note that the estimate obtained in the limit of an infinite disorder is close, though at the boundary of error bars, to the exponent $\frac{\beta}{\nu}=\frac{5}{36} \times \frac{3}{4} \simeq 0.10417 \ldots$ of percolation. This can be understood in the following way: in the limit $K_{1} \rightarrow+\infty$ and $K_{2} \rightarrow 0$, the random-bond Ising model is equivalent to a diluted Ising model at zero temperature. Long-range ferromagnetic order can exist only if the bonds percolate. The singularity of magnetization is due to the percolation transition. In the case of the random-bond Ising or Potts model, the percolation fixed point is known to be unstable. It manifests itself only by cross-over effects at small lattice sizes and strong disorder. In the simulations presented above, the calculation is performed exactly at the percolation fixed point. As a consequence, the exponent $\beta / \nu$ of the percolation fixed point is measured and no cross-over effect is expected.

To summarize, in the case of correlated disorder, the magnetic critical behavior that was previously reported at finite disorder strength is the same as in the limit of infinite strength. It is therefore governed by the correlated percolation fixed point [28]. As far as we are aware, only the value corresponding to the Ising model, i.e. $y=0$, has been reported to be $0.0527(4)$ assuming $\nu=1$ [29]. Even though no cross-over was observed at finite disorder strength, the stability of the fixed point remains to be shown. In the case of uncorrelated disorder, the percolation fixed point is unstable so that, at finite disorder, and therefore at finite temperature at the critical point, thermal fluctuations bring the system to the random fixed point if disorder is relevant, or to the pure fixed point otherwise. 


\section{Stability of the correlated percolation fixed point}

In the previous section, the calculations were performed exactly in the limit of an infinite disorder, i.e. at the (correlated) percolation point. In this section, the case of a large but finite disorder is considered. The partition function and the average square magnetization are expanded to first order around the (correlated) percolation point and the exponent of the first correction is estimated.

Even though the Ising case $q=2$ will be considered, we start with the Potts Hamiltonian. For a given disorder configuration $\left\{J_{i j}\right\}$, the partition function reads

$$
\mathcal{Z}\left[J_{i j}\right]=\sum_{\{s\}} e^{\sum_{(i, j)} J_{i j} \delta_{s_{i}, s_{j}}}=\sum_{\{s\}} \prod_{(i, j)}\left(u_{i j} \delta_{s_{i}, s_{j}}+1\right)
$$

where

$$
u_{i j}=e^{J_{i j}}-1
$$

Introducing $E_{1}$ (resp. $E_{2}$ ) the set of bonds for which $J_{i j}=J_{1}\left(\right.$ resp. $J_{i j}=J_{2}$ ), the partition function of the Potts model for a given coupling realization reads

$$
\mathcal{Z}\left[J_{i j}\right]=\sum_{\{s\}} \prod_{(i, j) \in E_{1}}\left(u_{1} \delta_{s_{i}, s_{j}}+1\right) \prod_{(i, j) \in E_{2}}\left(u_{2} \delta_{s_{i}, s_{j}}+1\right)
$$

On the square lattice, each coupling configuration $J_{i j}$ can be mapped onto a dual configuration $J_{i j}^{*}$ where

$$
u_{i j} u_{i j}^{*}=q
$$

with $u_{i j}^{*}=e^{J_{i j}^{*}}-1$. The duality transformation $J_{i j} \rightarrow J_{i j}^{*}$ exchanges strong and weak couplings and therefore maps the ferromagnetic phase onto the paramagnetic one. If a coupling configuration $J$ and its dual $J^{*}$ have the same probability, i.e. $\wp[J]=\wp\left[J^{*}\right]$, the model is self-dual and critical. In our case, this condition is satisfied when $J_{1}=J_{2}^{*}$ because the exchange of the couplings $J_{1}$ and $J_{2}$ is equivalent to a reversal of the polarization of the auxiliary Ashkin-Teller model. Along the critical line, the variable $u_{2}=q / u_{1}$ can be removed:

$$
\mathcal{Z}\left[J_{i j}\right]=u_{1}^{\left|E_{1}\right|} \sum_{\{s\}} \prod_{(i, j) \in E_{1}}\left(\delta_{s_{i}, s_{j}}+\frac{1}{u_{1}}\right) \prod_{(i, j) \in E_{2}}\left(\frac{q}{u_{1}} \delta_{s_{i}, s_{j}}+1\right)
$$

where $\left|E_{1}\right|$ is the number of couplings $J_{1}$. When $u_{1} \gg u_{2}=q / u_{1}$, an expansion in powers of $1 / u_{1}$ is easily obtained. To first order, the partition function is

$$
\mathcal{Z}\left[J_{i j}\right] \simeq u_{1}^{\left|E_{1}\right|} \sum_{\{s\}}\left[\prod_{(i, j) \in E_{1}} \delta_{s_{i}, s_{j}}+\frac{1}{u_{1}}\left(\sum_{(k, l) \in E_{1}} \prod_{\substack{(i, j) \\ \in E_{1} \backslash(k, l)}} \delta_{s_{i}, s_{j}}+q \sum_{(k, l) \in E_{2}} \prod_{\substack{(i, j) \\ \in E_{1} \cup(k, l)}} \delta_{s_{i}, s_{j}}\right)\right]
$$

Each term is proportional to the number of ways a graph can be decorated by Potts spins. Denoting $C(G)$ the number of connected clusters of a graph $G \subset E$, the partition function reads to first order

$$
\begin{aligned}
\mathcal{Z} & {\left[J_{i j}\right] \simeq u_{1}^{\left|E_{1}\right|}\left[q^{C\left(E_{1}\right)}+\frac{1}{u_{1}}\left(\sum_{(k, l) \in E_{1}} q^{C\left(E_{1} \backslash(k, l)\right)}+\sum_{(k, l) \notin E_{1}} q^{C\left(E_{1} \cup(k, l)\right)+1}\right)\right] } \\
& =u_{1}^{\left|E_{1}\right|} q^{C\left(E_{1}\right)}\left[1+\frac{1}{u_{1}}\left(\sum_{(k, l) \in E_{1}} q^{C\left(E_{1} \backslash(k, l)\right)-C\left(E_{1}\right)}+\sum_{(k, l) \notin E_{1}} q^{C\left(E_{1} \cup(k, l)\right)+1-C\left(E_{1}\right)}\right)\right]
\end{aligned}
$$


The quantity $C\left(E_{1} \backslash(k, l)\right)-C\left(E_{1}\right)$ vanishes for all bonds $(k, l)$ of $E_{1}$ that do not disconnect a cluster into two unconnected parts when they are removed. It takes the value +1 otherwise, i.e. for the so-called red bonds, or bridges, of the graph $E_{1}$. The quantity $C\left(E_{1} \cup(k, l)\right)-C\left(E_{1}\right)$ vanishes if adding a bond $(k, l)$ does not change the number of clusters, i.e. if the vertices $k$ and $l$ are connected by some path on the graph $E_{1}$. It takes the value -1 otherwise, i.e. when the vertices $k$ and $l$ belong to different clusters of the graph $E_{1}$. Numerically, the first quantity is determined by identifying the red bonds with the Tarjan algorithm [30]. The second quantity is computed using a labelling of the clusters during their identification.

The same expansion is now written for the average square magnetization of the Ising model, i.e. the case $q=2$ :

$$
\left\langle M^{2}\right\rangle=\frac{1}{\mathcal{Z}\left[J_{i j}\right]} u_{1}^{\left|E_{1}\right|} \sum_{\{s\}}\left(\sum_{i} s_{i}\right)^{2} \prod_{(i, j) \in E_{1}}\left(\delta_{s_{i}, s_{j}}+\frac{1}{u_{1}}\right) \prod_{(i, j) \in E_{2}}\left(\frac{q}{u_{1}} \delta_{s_{i}, s_{j}}+1\right)
$$

To first order and performing the average over the spin decorations of the clusters, the square magnetization reads

$$
\begin{aligned}
\left\langle M^{2}\right\rangle & \simeq\left\langle M^{2}\right\rangle_{E_{1}} \\
& +\frac{q^{-C\left(E_{1}\right)}}{u_{1}}\left(\sum_{(k, l) \in E_{1}} q^{C\left(E_{1} \backslash(k, l)\right)}\left\langle M^{2}\right\rangle_{E_{1} \backslash(k, l)}+q \sum_{(k, l) \in E_{2}} q^{C\left(E_{1} \cup(k, l)\right)}\left\langle M^{2}\right\rangle_{E_{1} \cup(k, l)}\right) \\
& -\frac{1}{u_{1}}\left\langle M^{2}\right\rangle_{E_{1}}\left(\sum_{(k, l) \in E_{1}} q^{C\left(E_{1} \backslash(k, l)\right)-C\left(E_{1}\right)}+\sum_{(k, l) \notin E_{1}} q^{C\left(E_{1} \cup(k, l)\right)+1-C\left(E_{1}\right)}\right)
\end{aligned}
$$

where $\left\langle M^{2}\right\rangle_{E_{1}}$ denotes a calculation at zeroth-order in $1 / u_{1}$ for the graph $E_{1}$ along the lines presented after Eq. 18. Finally, we get

$$
\begin{aligned}
\left\langle M^{2}\right\rangle \simeq\left\langle M^{2}\right\rangle_{E_{1}} & +\frac{1}{u_{1}} \sum_{(k, l) \in E_{1}} q^{C\left(E_{1} \backslash(k, l)\right)-C\left(E_{1}\right)}\left(\left\langle M^{2}\right\rangle_{E_{1} \backslash(k, l)}-\left\langle M^{2}\right\rangle_{E_{1}}\right) \\
& +\frac{1}{u_{1}} \sum_{(k, l) \in E_{2}} q^{C\left(E_{1} \cup(k, l)\right)+1-C\left(E_{1}\right)}\left(\left\langle M^{2}\right\rangle_{E_{1} \cup(k, l)}-\left\langle M^{2}\right\rangle_{E_{1}}\right)
\end{aligned}
$$

The only strong couplings $J_{k l}$, i.e. $(k, l) \in E_{1}$, contributing to the first sum are the red bonds of the graph $E_{1}$. Their removal provokes the splitting of a cluster of strong couplings into two unconnected clusters so, as discussed before, $C\left(E_{1} \backslash(k, l)\right)$ $C\left(E_{1}\right)=1$. The change in the total square magnetization, $\left\langle M^{2}\right\rangle_{E_{1} \backslash(k, l)}-\left\langle M^{2}\right\rangle_{E_{1}}$, is computed numerically simply by first removing the red bond and then relabelling the cluster starting at one edge of the bond. The only weak couplings $J_{k l}$, i.e. $(k, l) \notin E_{1}$, contributing to the second sum of Eq. 31 are those for which a different cluster is found at the two edges. Adding this bond leads to the merging of the two clusters so that $C\left(E_{1} \cup(k, l)\right)+1-C\left(E_{1}\right)=0$. The calculation of the change in the total square magnetization, $\left\langle M^{2}\right\rangle_{E_{1} \cup(k, l)}-\left\langle M^{2}\right\rangle_{E_{1}}$, only requires a minimal computational effort if the sizes of the different clusters were stored. After performing the average over disorder configurations, the average square magnetization can be put in the form

$$
{\overline{\left\langle M^{2}\right\rangle_{u_{1}}}}={\overline{\left\langle M^{2}\right\rangle_{\infty}}}_{\infty}\left[1+\frac{1}{u_{1}}\left(q C_{1}+C_{2}\right)\right]
$$

where the two correction terms are

$$
C_{1}=\frac{\overline{\sum_{(k, l) \in E_{1}}\left[\left\langle M^{2}\right\rangle_{E_{1} \backslash(k, l)}-\left\langle M^{2}\right\rangle_{E_{1}}\right]_{\infty}}}{\overline{\left\langle M^{2}\right\rangle_{\infty}}}
$$



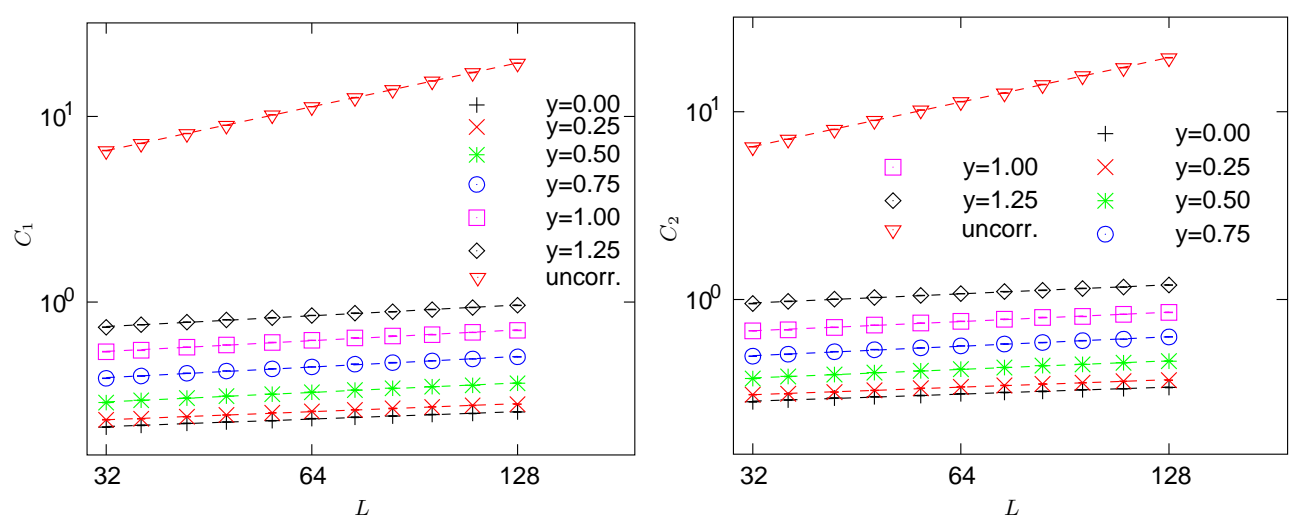

Fig. 3. Finite-size scaling of the two contributions $C_{1}$ (left) and $C_{2}$ (right) to the first-order correction to the square magnetization of the 2D Ising model in the limit of infinite disorder. The different symbols correspond to the Monte Carlo data for different disorder correlations. The parameter $y$ is given in the legend. The case of uncorrelated disorder is also plotted and denoted 'uncorr.' in the legend. Errors bars are plotted. The dashed lines are the power-law fits.

and

$$
C_{2}=\frac{\overline{\sum_{(k, l) \notin E_{1}}\left[\left\langle M^{2}\right\rangle_{E_{1} \cup(k, l)}-\left\langle M^{2}\right\rangle_{E_{1}}\right]_{\infty}}}{\overline{\left\langle M^{2}\right\rangle_{\infty}}}
$$

The two correction terms $C_{1}$ and $C_{2}$ were computed for 100.000 graphs $E_{1}$ corresponding to disorder configurations $J_{i j}$, and therefore to polarization configurations of the auxiliary Ashkin-Teller model. The data are plotted on figure 3 versus the lattice size $L$. Nice power laws are observed. Slightly larger exponents are obtained for $C_{1}$ than for $C_{2}$ (see the estimates in table 1). In the case of uncorrelated disorder, this exponent is $\omega \simeq 0.79$. In contrast, for correlated disorder, the exponent is much smaller but positive. There are two important consequences: first, the correction terms grow with the lattice size which implies that they will eventually become dominant. The correlated percolation fixed point is therefore unstable. Second, the cross-over length $L^{*}$ at which the correction becomes of order $\mathcal{O}(1)$ for a given $u_{1}$ is much smaller in the uncorrelated case. For $L=32$ for instance, $C_{1} \simeq 6.55$ for uncorrelated disorder while $C_{1} \simeq 0.73$ for $y=1.25$. Assuming a correction exponent $\omega \simeq 0.2, C_{1}$ will be equal to 6.55 only at a lattice size $L \simeq 2.3 .10^{6}$ ! The situation is even worse in the case $y=0$ for which a lattice size $L \simeq 2.5 .10^{12}$ is needed for $C_{1}$ to reach the value 6.55 . Such huge lattice sizes cannot be reached by Monte Carlo simulations. This explains why the cross-over was not observed in previous Monte Carlo simulations at finite disorder strength.

\section{Conclusion}

By performing calculations explicitly in the limit of an infinite disorder strength, it was shown that the magnetic scaling dimensions previously reported for the random Potts model with slowly-decaying disorder correlations correspond to the correlated percolation fixed point. The analysis of the first-order corrections at finite disorder strength revealed that this fixed point is unstable. However, the cross-over length associated to these corrections is found to be much larger, by several orders of magnitude, than in the uncorrelated case. Therefore, the true critical behavior cannot be 
reached by Monte Carlo simulations. Nevertheless, it is interesting that this model reproduces features of an infinite-disorder fixed point even though the latter is not stable in this case. One may hope that it will help in a better understanding of the stability of such fixed points.

\section{Acknowledgments}

The author would like to congratulate Wolfhard Janke at the occasion of his 60th birthday and gratefully thanks the organizers of the workshop dedicated to this event.

\section{References}

1. Y. Imry, and M. Wortis Phys. Rev. B 193581 (1979).

2. M. Aizenman, and J. Wehr Phys. Rev. Lett. 622503 (1989).

3. A.B. Harris J. Phys. C: Solid State Phys. 71671 (1974).

4. J.L. Cardy, and J.L. Jacobsen Phys. Rev. Lett. 794063 (1997).

5. J.L. Jacobsen, and J.L. Cardy Nucl. Phys. B 515701 (1998).

6. C. Chatelain, and B. Berche Nucl. Phys. B 572626 (2000).

7. A.W.W. Ludwig Nucl. Phys. B 28597 (1987).

8. A.W.W. Ludwig, and J.L. Cardy Nucl. Phys. B 285687 (1987).

9. Vl.S. Dotsenko, M. Picco, and P. Pujol Phys. Lett. B 347113 (1995).

10. Vl.S. Dotsenko, M. Picco, and P. Pujol Nucl. Phys. B 455701 (1995).

11. L. Schwenger, K. Budde, C. Voges, and H. Pfnür Phys. Rev. Lett. 73296 (1994).

12. A. Weinrib, and B.I. Halperin Phys. Rev. B 27413 (1983).

13. H. G. Ballesteros, and G. Parisi Phys. Rev. B 6012912 (1999).

14. D. Ivaneyko, B. Berche, Y. Holovatch, and J. Ilnytskyi Physica A 3874497 (2008).

15. M. Dudka, A.A. Fedorenko, V. Blavatska, and Y. Holovatch Phys. Rev. B 93, 224422 (2016).

16. C. Chatelain Eur. Phys. Lett. 10266007 (2013).

17. C. Chatelain Phys. Rev. E 89032105 (2014).

18. R.B. Griffiths Phys. Rev. Lett. 23, 17 (1969).

19. T. Vojta J. Phys. A 39, R143 (2006).

20. B.M. McCoy and T.T. Wu Phys. Rev. 176631 (1968).

21. B.M. McCoy and T.T. Wu Phys. Rev. 188982 (1969).

22. D.S. Fisher Phys. Rev. B 516411 (1995).

23. M. Schwartz, and A. Soffer Phys. Rev. Lett. 552499 (1985).

24. T. Senthil and S.N. Majumdar Phys. Rev. Lett. 763001 (1996).

25. R. B. Potts Math. Proc. Camb. Phil. Soc. 48106 (1952).

26. J. Ashkin et E. Teller Phys. Rev. 64178 (1943).

27. C. Fan Phys. Lett. A 39136 (1972).

28. A. Coniglio, and A. Fierro Encyclopedia of Complexity and Systems Science, Part 3, 1596-1615, Springer New York (2009).

29. W. Janke, and A. Schakel Braz. J. Phys. 36708 (2006).

30. R. Tarjan Information Processing Letters 2160 (1974). 